\title{
Lithium in cool magnetic CP stars: Some new results of observations, using CAT (ESO) 2.6m (CrAO), (NOT) La Palma telescopes.
}

\author{
N. Polosukhina ${ }^{1}$, D.Kurtz ${ }^{2}$, M. Hack ${ }^{3}$, P. North ${ }^{4}$, I. Ilyin ${ }^{5}$, J. Zverko \\ ${ }^{1}$ Crimean Astrophysical Observatory Nauchny, Crimea, Ukraine \\ ${ }^{2}$ University of Cape Town, Rondebosh 7700, South Africa \\ ${ }^{3}$ Department of Astronomy, Trieste University, Italy \\ ${ }^{4}$ Institut d'Astronomie de l'Universitè de Lausanne, CH 1290,Switzerland \\ ${ }^{5}$ University of Oulu, P.O. Box 3000, 90401 Oulu, Finland \\ ${ }^{6}$ Astronomical Institute of the Slovak Academy of Science, 05960 \\ Tatranska Lomnica, The Slovak Republic
}

\section{Introduction}

Lithium in cool magnetic CP stars in still poorly studied and estimations of the $\mathrm{Li}$ abundance in these stars are scarce. There is some evidence of variability of the LiI $6708 \AA$ line, but this variability has not been studied systematicaly. Even the identification of the $6708 \AA$ line with the LiI resonance doublet is still in doubt. This problem is important in the broader context of the Li abundance in various types of stars, as well as for deeper undersfanding of the magnetic star phonomenon itself. The reason for fhis is that the $\mathrm{Li}$ abundance in very sensitive to evolutionary status of the stars and their properties, such as the character and intensity of mixing processes.

\section{Observations}

The majority of the observations presented here were made by P. North with the European Southern Observatory (ESO) Coudé Auxiliary Telescope (CAT). The Coudé Echelle Spectrograph was used with resolving power $\mathrm{R}=3 \mathrm{D} 100000$ and the $\mathrm{S} / \mathrm{N}$ ratio for an individual spectrogram better than 100 per pixel in $1 \sigma$ level. The detector was the ESO CCD 34 with 2048 pixels along the dispersion. A Thorium-Argon lamp was used for the wavelength calibrations with accuracy better than $0.3 \mathrm{~km} / \mathrm{s}$. The wavelength range observed was $6675-6735$ $\AA$. The spectra were reduced by PN using the standard IRAF procedures. The observations made at the $\mathrm{CrAO}$ were a part of a long-term program, some results of which were published in Hack et al.(1997) and North et al.(1998). A Coudé spectrograph of the Shajn 2.6-m telescope was used; it is equipped with a CCD camera with a red-sensitive detector with a $600 \times 400$ pixel array. The linear dispersion is $2.5 \AA / \mathrm{mm}$ in the region of $6708 \AA$ and $\mathrm{R}=3 \mathrm{D} 65000$. 

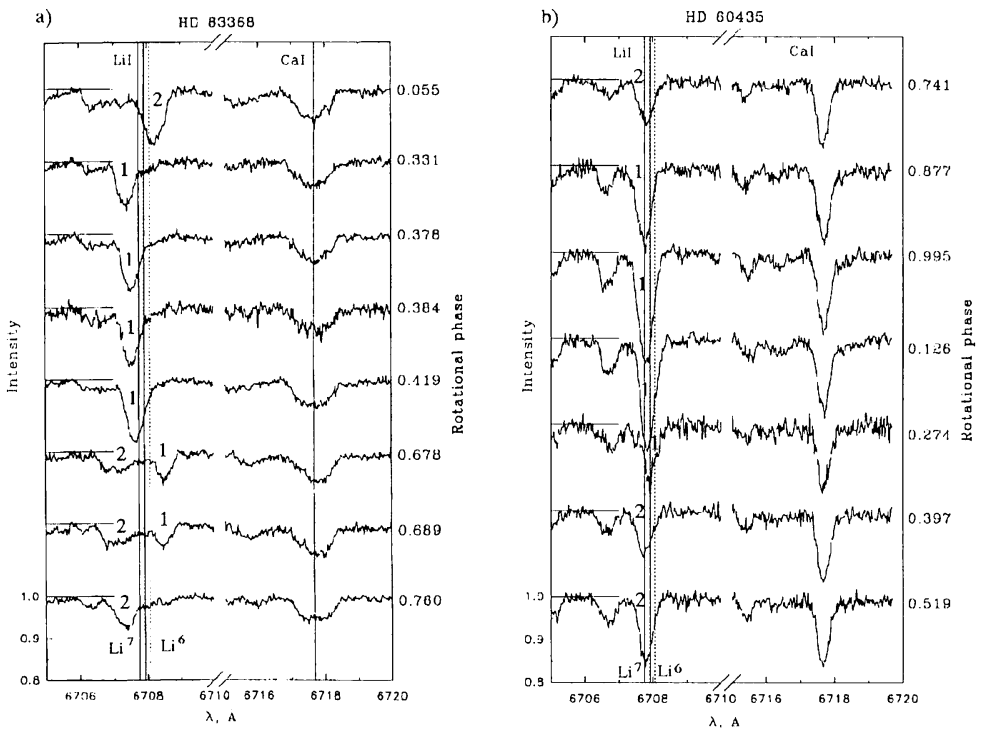

Figure 1. The spectra of ro Ap stars HD 83368 and HD 60435 in LiI $6708 \AA$ region in residual intensity scale. At the left side of each spectrum the position of the continium is shown by line. At the right side is indicated rotation phases. The doppler components of Li line $6708 \AA$ are marked 1,2 . 

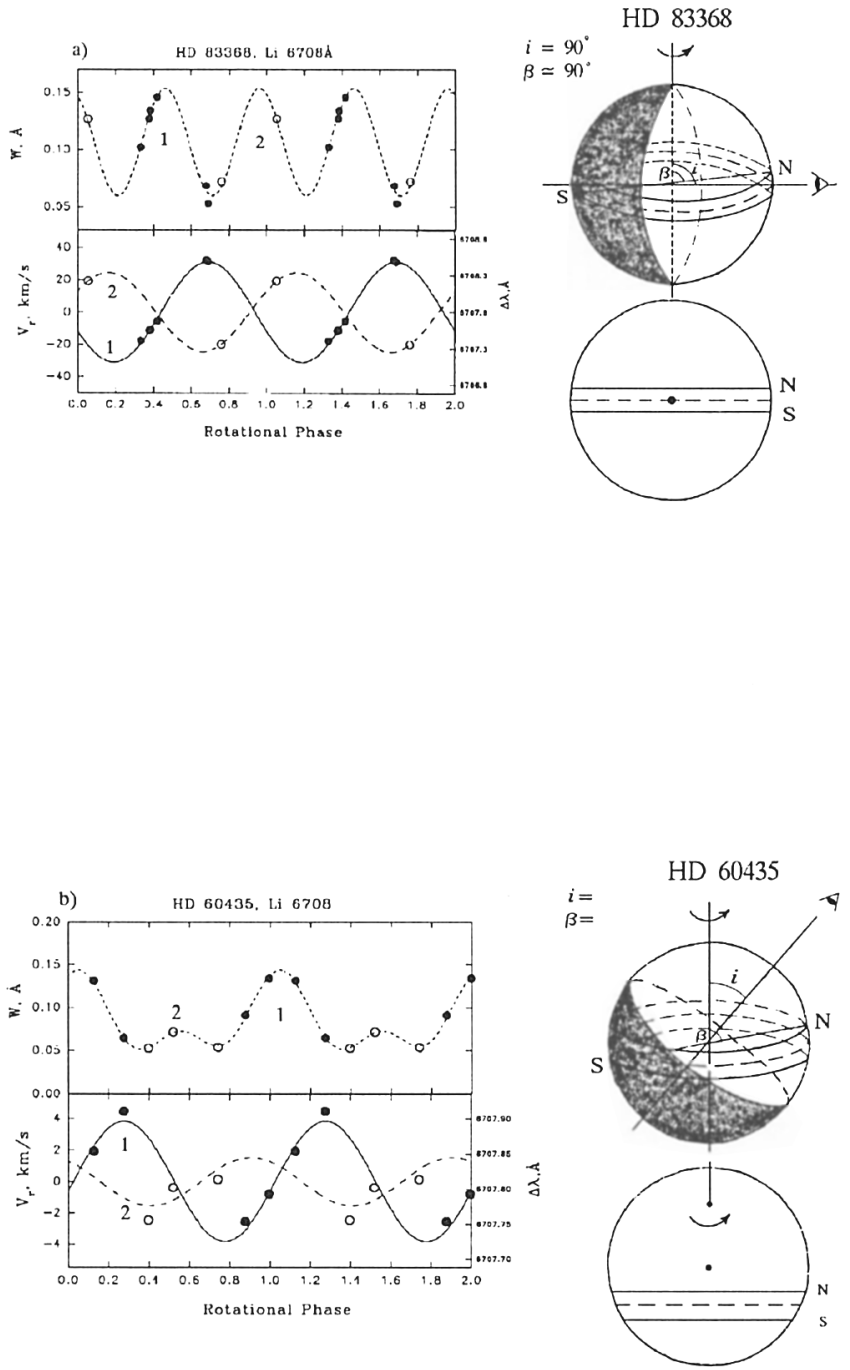

Figure 2. Variations of the equivalent widths EW(top) and of the radial velocities $\mathrm{Vr}$ of $\mathrm{Li}$ line $6708 \AA$ (bottom). The black dots indicate the spot 1 , and circles - spot 2 . At the right side is shown model oblique rotator for every star. $\mathrm{N}, \mathrm{S}$ - poles of magnetic dipole are coincided with $\mathrm{Li}$ spots $1,2$. 
33 Lib

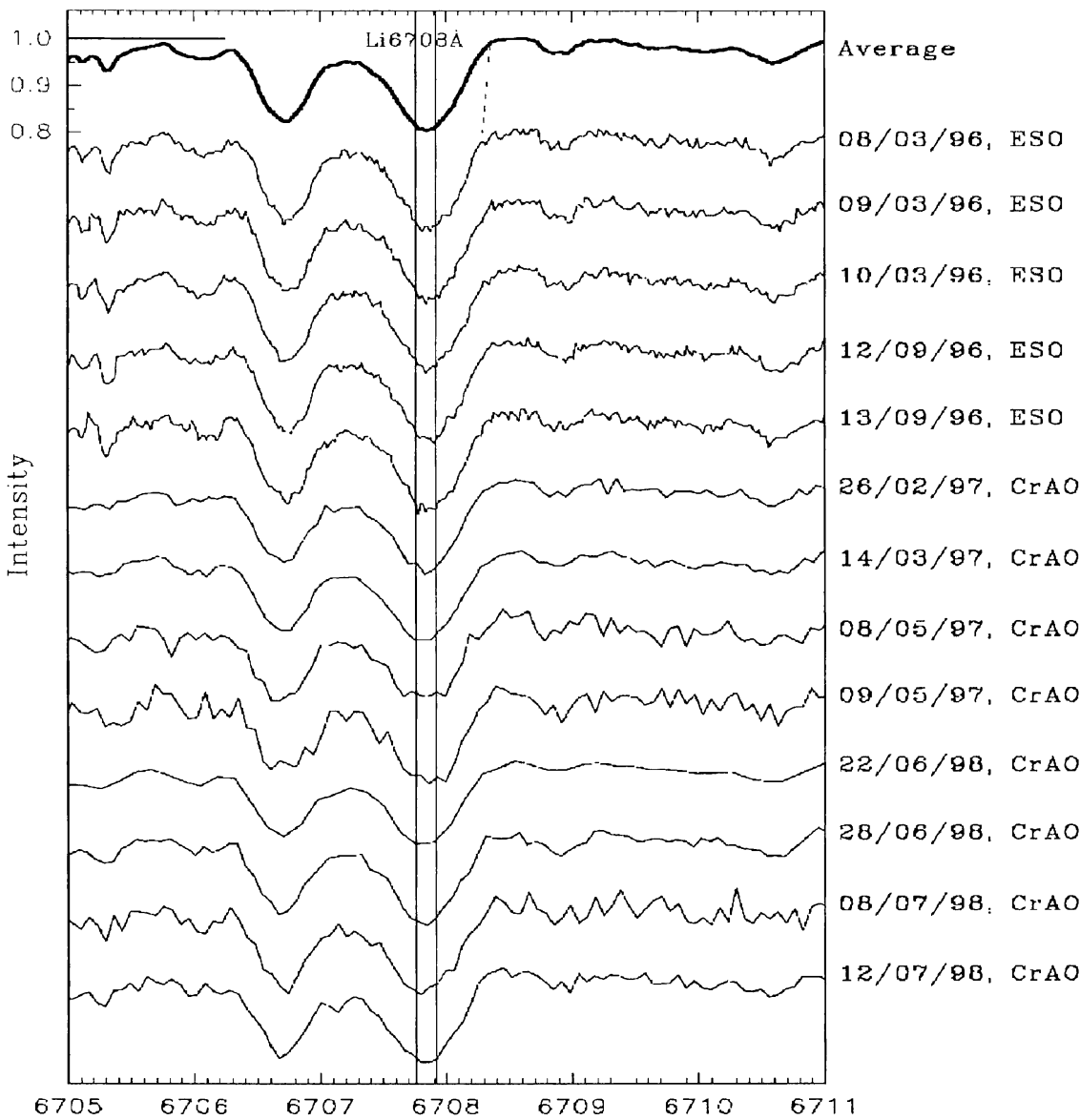

$\lambda, \dot{A}$

Figure 3. The spectra $33 \mathrm{Lib}$ in LiI 6708 region in residnal intensity scale. Thick line is average spectrum. At the right side is indicated telescopes $\mathrm{ESO}(\mathrm{CAT})$ and $\mathrm{CRAO}(2.6 \mathrm{~m})$ are used for observations, and model oblique rotator for groupe 3 stars. 
A typical $\mathrm{S} / \mathrm{N}$ ratio is better than 100 . The wavelength range was 6690 $9730 \AA$. The spectra were reduced by DS using the package of S.Sergeev. The observations with the Nordic Optical Telescope (NOT) on La Palma, Spain, were carried out with the SOFIN echelle spectrograph (Tuominen, 1992) and two CCD cameras, yielding $R=3 D 80000$ and 160000 in the $5500-8500 \AA$ range and the $\mathrm{S} / \mathrm{N}$ ratio $90-300$ per pixel. A detailed description of the observations and the discussion of the line identification problems may be found in Hack et al. (1997), North et al. (1998), and Zverko et al. (1998).

\section{Results and Conclusion.}

The resonance LiI $6708 \AA$ line was observed in spectra of twelve program Ap-CPstars. The behaviour of the line $6708 \AA$ permits us to distingnish four groupes of stars:

* groupe 1 - the line shows great variability of profile Li line and "W", "RV" with rotation phase (Fig 1a,b). This behaviour can be explained by the presence of two Li spots on the star's surface.=20 (HD 83386, HD 60435) (Fig $2 \mathrm{a}, \mathrm{b})$

* group 2 - the line is variable, but the observations are too sparse to make a conclusion about the variability nature. (HD 188041, $\beta \mathrm{CrB}$ )

* group 3 - the line is strong, but nonvariable. (33 Lib, HD 134214, HD 166473) (Fig 3a)

* group 4 - the line was not detected ( HD 42659, HD 80316, HD 118022 and $\mathrm{HD} 128898$ )

- The discovery of LiI-spots in HD 83368 and in 60435 is the first indication of spottiness in lithium distribution on surface of some cool magnetic $=20$ CP-stars.

- A good correlation between the positions of the spots, magnetic poles, and oscillation phenomena (HD 83368) indicates possible connections between the magnetic fields configuration and the local structure of star's atmosphere. (Polosukhina N. et al, 1999)

- We explaned the behaviour of $6708 \AA$ line using the model of the "spotted" oblique rotator, with different parameters for each star(Fig 2a,b). The anles " $i$ " and " $\beta$ " for different stars defermine the visibility of magnetic poles and spots, consequently, behaviour lithium line $6708 \AA$. HD 83368 is unique star, the lithium are situated at the poles of the magnetic dipole. The magnetic dipole is placed hear equator's plane. The angles $i \simeq 90^{\circ}$ and $\beta \simeq 90^{\circ}$ too. $=20$ In the case of constancy of the line $6708 \AA$ (group 3 ), observer sees spot near rotation pole $i \leq 30^{\circ}, \beta \leq 30^{\circ}$ (Fig $3 \mathrm{~b}$ )

- The results of observations in Li I $6708 \AA$ line and discovery Li spots on magnetic poles are good argee with prediction Babel theory, concern ambipolar diffusion of hydrogen in CP-stars with dipole structure of magnetic field (Babel J., Michaud G., 1991). 


\section{References}

Hack M., Polosukhina N., Malanushenko V., Castelli F., 1997, A\&A 319, 637

North P., Polosukhina N., Malanushenko V., Hack M., 1998, A\&A 333, 644

Zverko J., Žižňovský J., North P., 1998, Contrib. Astron.Obs.Skalnaté Pleso 28, 109

Polosukhina N., North P., D.Kurtz D., Hack M., Zverko J., Ilyin I., Shakhovskoy D. 1999, A\&A 351, 283-291

Babel J., Michaud G., 1991 Ap.J., 366, 560 